\title{
DISTRIBUTED TRAFFIC COMPLEXITY MANAGEMENT BY PRESERVING TRAJECTORY FLEXIBILITY
}

\author{
Husni Idris, Robert Vivona, Jose-Luis Garcia-Chico, L-3 Communications, Billerica, MA \\ David Wing, NASA Langley Research Center, Hampton, VA
}

\begin{abstract}
In order to handle the expected increase in air traffic volume, the next generation air transportation system is moving towards a distributed control architecture, in which groundbased service providers such as controllers and traffic managers and air-based users such as pilots share responsibility for aircraft trajectory generation and management. This paper presents preliminary research investigating a distributed trajectoryoriented approach to manage traffic complexity, based on preserving trajectory flexibility. The underlying hypotheses are that preserving trajectory flexibility autonomously by aircraft naturally achieves the aggregate objective of avoiding excessive traffic complexity, and that trajectory flexibility is increased by collaboratively minimizing trajectory constraints without jeopardizing the intended air traffic management objectives. This paper presents an analytical framework in which flexibility is defined in terms of robustness and adaptability to disturbances and preliminary metrics are proposed that can be used to preserve trajectory flexibility. The hypothesized impacts are illustrated through analyzing a trajectory solution space in a simple scenario with only speed as a degree of freedom, and in constraint situations involving meeting multiple times of arrival and resolving conflicts.
\end{abstract}

\section{Introduction}

The Next Generation Air Transportation System (NextGen) is expected to receive up to three times the current traffic demand by the year 2025 [1]. In order to handle the expected increase in air traffic NextGen will introduce key transformations in Air Traffic Management (ATM), three examples of which are: net-enabled information access, performance-based services, and aircraft trajectorybased operations [1]. Net-enabled information access will substantially increase information sharing and awareness of system operations among users and service providers. For example, the emerging Automatic Dependent SurveillanceBroadcast (ADS-B) technology enables sharing of aircraft-based position and intent information among airborne and ground-based agents. Performance-based services will make access to National Airspace System (NAS) resources, such as runways and airspace volumes, dependent on aircraft equipage and capability. Trajectory-based operations will require aircraft to precisely follow custom-made four dimensional (4D) trajectories consisting of a specified path and along-path time conformance requirements. Such accurate trajectories ensure separation and optimize traffic flow management over different time horizons.

These capabilities enable a more optimal allocation of functions among the air traffic system agents [2]. One such allocation scheme proposes moving the ATM system towards a distributed control architecture $[3,4]$, which delegates to the pilot more authority over the aircraft trajectory; currently this authority resides mainly with the ground-based controller except in emergency situations. The premise is to mitigate the controller workload as a constraint against increasing airspace capacity, because introducing more traffic introduces additional responsible decision makers (pilots) enabled by advanced sensor, communication, and decision support technologies.

While the architecture of the ATM system becomes more distributed, its goal remains to achieve objectives such as maintaining safety and efficiency at acceptable levels. The ability of a distributed control architecture to satisfy these objectives has important implications on the new role of centralized control, taking on higher level supervisory functions such as monitoring and intervention, as opposed to lower level active control, thus enabling capacity gains and cost savings. To this end it is critical to design the distributed architecture with appropriate elements that ensure individual aircraft actions achieve the overall ATM objectives. 
Prior research on distributed ATM focused on the investigation of sharing the primary function of separation assurance between pilots and controllers. A number of algorithms for conflict resolution in a distributed environment were reported [5-7]. To assist the pilot, automation such as the Autonomous Operations Planner (AOP) is designed to provide conflict detection and resolution advisories in the cockpit [8], using genetic algorithms to resolve conflicts between aircraft pairs $[9,10]$. Early experiments of mixed distributed and centralized separation assurance showed promising effects on workload and efficiency $[11,12,13]$.

This paper presents preliminary research investigating two functions for the distributed ATM system that have been newly proposed in Idris et al. [14]: A trajectory flexibility preservation function and a trajectory constraint minimization function. Trajectory flexibility preservation enables an aircraft to plan its trajectory such that it preserves a requisite level of maneuvering flexibility in accommodating disturbances, caused for example by other traffic and weather activity. Trajectory constraint minimization enables ground-based agents, in collaboration with air-based agents, to impose just-enough constraints on trajectories to achieve ATM objectives, such as separation assurance and flow management. The concept hypothesizes that by each aircraft autonomously preserving its own trajectory flexibility, aggregate system objectives, such as maintaining acceptable traffic complexity (complexity defined as proneness to compromising safety), are naturally achieved. It also hypothesizes that minimizing the constraints imposed on a trajectory, without jeopardizing the intended ATM objectives, increases its flexibility.

In this paper, the second section reviews from [14] how trajectory flexibility preservation and constraint minimization operate in a distributed control architecture that includes self separation. The underlying hypotheses are illustrated through hypothetical scenarios involving conflict resolution and flow management. The third section describes an analytical framework for defining metrics and developing methods to preserve trajectory flexibility and minimize its constraints. In this framework flexibility is defined in terms of robustness and adaptability to disturbances. The forth section describes a simple analytical example in the context of which specific metrics are proposed. The example demonstrates qualitatively the use of the metrics for selecting flexible paths in simple constraint situations involving meeting multiple times of arrival as constraints and accommodating a conflict as a disturbance, using speed as a degree of freedom. Finally, concluding remarks and future research are discussed.

\section{Concept of Distributed Trajectory Flexibility Preservation and Constraint Minimization}

This concept centers around three key functions: separation assurance, flexibility preservation and constraint minimization. Each function, the relationships between them, and their impact on NAS performance indicators such as capacity and complexity, are described [14].

\section{Separation Assurance}

Separation assurance is the central function of air traffic control, taking in its time horizon and for safety reasons priority over other functions such as implementing traffic flow management initiatives. In centralized control, separation assurance is the responsibility of the air traffic controller who ensures the minimum separation requirements between aircraft within an airspace volume. In a distributed control architecture, each aircraft (i.e. pilot/automation system) is responsible for maintaining separation from surrounding traffic. Pilots are assisted in conflict detection and resolution by cockpit automation, such as the AOP system, thus maintaining their workload at an acceptable level [8]. The allocation of separation assurance tasks to pilots reduces traffic complexity from a centralized perspective, which represents controller workload and proneness to separation violation errors $[15,16]$, because the controller is relieved from active separation assurance for selfseparating aircraft. In addition, a notion of distributed/automated traffic complexity is introduced representing proneness to separation violation errors in the new distributed/automated environment. For example, Riley et al. analyzed a pilot's perception of traffic complexity in airborne conflict resolution scenarios [17]. Therefore, traffic complexity may be represented and mitigated differently in a distributed/automated-control 
environment than in the centralized/human-control environment. The premise of the distributed control architecture is accommodating more traffic because the NAS capacity to assure separation is increased through the participation of pilots. Furthermore, as the traffic level increases, the NAS capacity for separation assurance increases, because introducing more traffic introduces more pilot decision makers for self separating aircraft, adding scalability of capacity with demand.

\section{Trajectory Flexibility Preservation}

Trajectory flexibility preservation is envisioned as an airborne function. It plans the trajectory in a manner that affords the aircraft sufficient flexibility in accommodating disturbances that stem, for example, from other traffic or from weather activity. Flexibility preservation complements separation assurance both within the conflict resolution horizon and outside it within an extended flexibility planning horizon [14].

In the conflict resolution horizon, flexibility is used to select from many conflict resolution solutions one that affords the aircraft more flexibility, for example to adapt to unexpected intruder traffic behavior. One example is shown in Figure 1, where two conflicts are predicted between two unrelated pairs of aircraft in the left side of the figure. If the two aircraft labeled "ownship" maneuvered to resolve their respective predicted

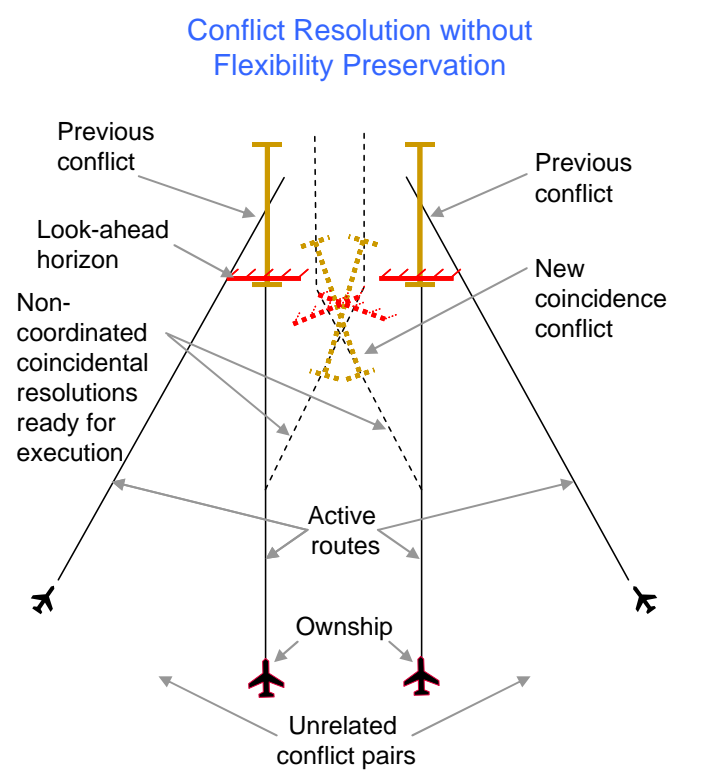

conflict, without coordination, a new coincidental conflict may arise between them as shown by dotted lines. Although flexibility preservation does not explicitly coordinate between the two aircraft, it assists each ownship in reducing the risk of conflict due to the unpredicted behavior of the surrounding traffic, thus resulting in implicit coordination. Hence with this function, each aircraft may select a more flexible trajectory anticipating the potential maneuver of the other aircraft and maneuvering away from it as shown in the right side of Figure 1.

Beyond the conflict resolution horizon, the flexibility preservation function plans the aircraft trajectory to minimize its exposure to disturbances such as weather cells and dense traffic areas. In this longer horizon the possibility of loss of separation is not time critical because conflict prediction is rather inaccurate and does not warrant conflict resolution. However, flexibility preservation reduces the probability of future conflicts, by minimizing the aircraft's exposure to weather cells and dense traffic areas. It is hypothesized that flexibility preservation naturally produces traffic situations that are less complex than without its application. Figure 2 depicts an example involving aircraft maneuvering between convective weather cells. On the left side each aircraft, while planning its trajectory questions whether it should modify its trajectory to increase a flexibility metric. If the aircraft proceeded along their depicted headings a complex traffic situation arises causing excessive

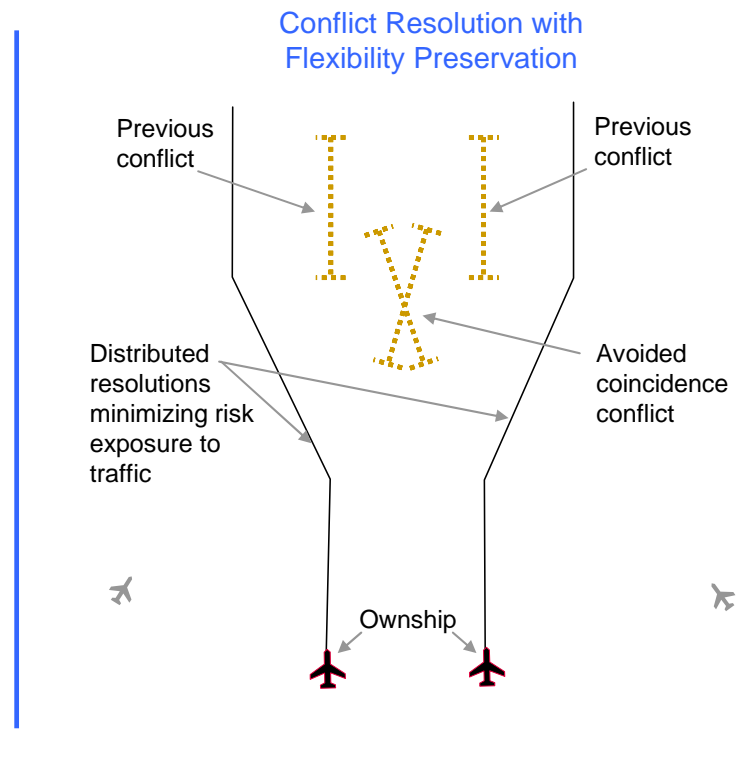

Figure 1 Flexibility Preservation Avoiding Coincidence Conflict 
Applicability of Trajectory Flexibility Prediction

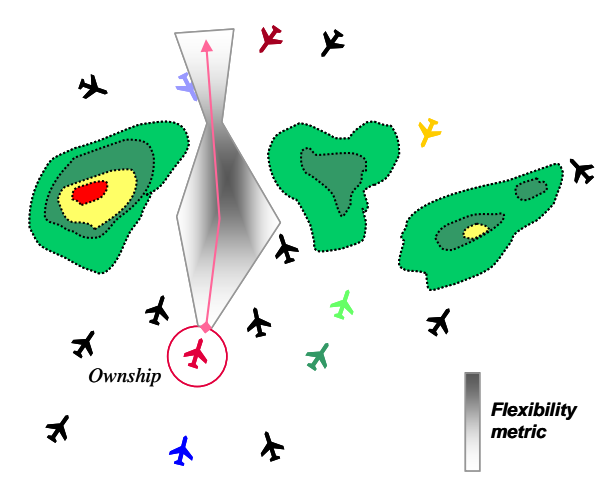

Airborne flexibility function will question:

Do I have enough flexibility to safely proceed?

Can I modify my trajectory to increase my flexibility?

Do I need to avoid this airspace entirely and replan?
Trajectories Designed to Preserve Flexibility

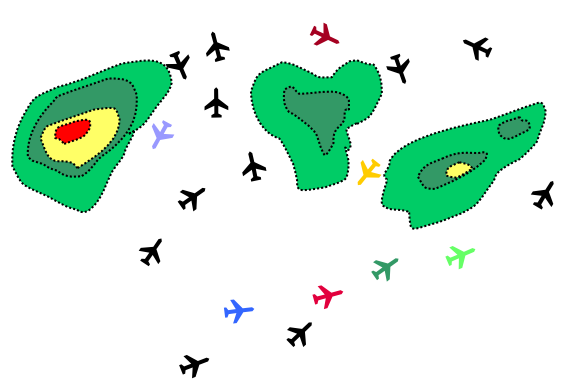

Hypothesis:

If all aircraft apply flexibility preservation function, complexity automatically will be reduced

Figure 2 Flexibility Preservation Avoiding Weather Cells and Congestion

congestion and a high potential conflict rate in the airspace between the weather cells. On the other hand, the right side of the figure displays a more structured and streamlined traffic pattern that is hypothesized to result if each aircraft increased its flexibility - by limiting its exposure to the other traffic and the weather cells. The workload of both the pilot (using automation tools to perform self separation) and the ground controller (performing flow management) are reduced, because the traffic is structured and the chance of conflict is reduced.

\section{Trajectory Constraint Minimization}

Trajectory constraint minimization is envisioned as primarily a ground-based function, with a possible collaboration role for the pilot. Constraint minimization assists a traffic manager to impose just enough constraints on the aircraft to meet the intended ATM objective. For example, if a single required time of arrival (RTA) at a specified fix will sufficiently meter the traffic flow, multiple RTAs per aircraft are deemed too excessive and hence candidate for relaxation. Constraint minimization enables more efficient utilization of NAS resources; but also increases the pilot's ability to maneuver freely with fewer constraints in order to accommodate disturbances. Therefore, while the traffic manager (assisted by automation such as the Center TRACON Automation System [18]), has the ability to monitor a large number of aircraft and achieve ATM objectives, the pilot may negotiate constraint reduction from the cockpit perspective. For example, the airborne automation may determine that the aircraft cannot meet all its constraints with enough flexibility, and hence the pilot may provide information to help the traffic manager relax some constraints.

Figure 3 demonstrates the hypothesized impact of constraint minimization on trajectory flexibility preservation and hence traffic complexity. Aircraft 'A' plans its trajectory to resolve a predicted conflict with aircraft ' $\mathrm{B}$ ' and to meet an RTA at a downstream fix. The RTA tolerance initially allows aircraft ' $A$ ' to avoid the predicted conflict only by path stretching to the left, with expected times of arrival (ETA) at the fix that lie within the RTA tolerance (left side of figure). These trajectories expose aircraft ' $A$ ' to disturbances from nearby traffic (Aircraft $\mathrm{C}$ and $\mathrm{D}$ ) and an inclement weather system, and they would increase its contribution to traffic complexity. With this information, the traffic manager relaxes the RTA constraint by increasing its tolerance as shown in the right side of the figure, having determined that the RTA's intended ATM objectives can still be met sufficiently. With the extended tolerance, aircraft 'A' has more maneuvering options to avoid the predicted conflict to the right and select a more flexible trajectory with less exposure to the weather and traffic, thus reducing the aircraft contribution to traffic complexity. The aircraft is also able to meet its RTA constraint more reliably and hence achieve the intended ATM objectives. 


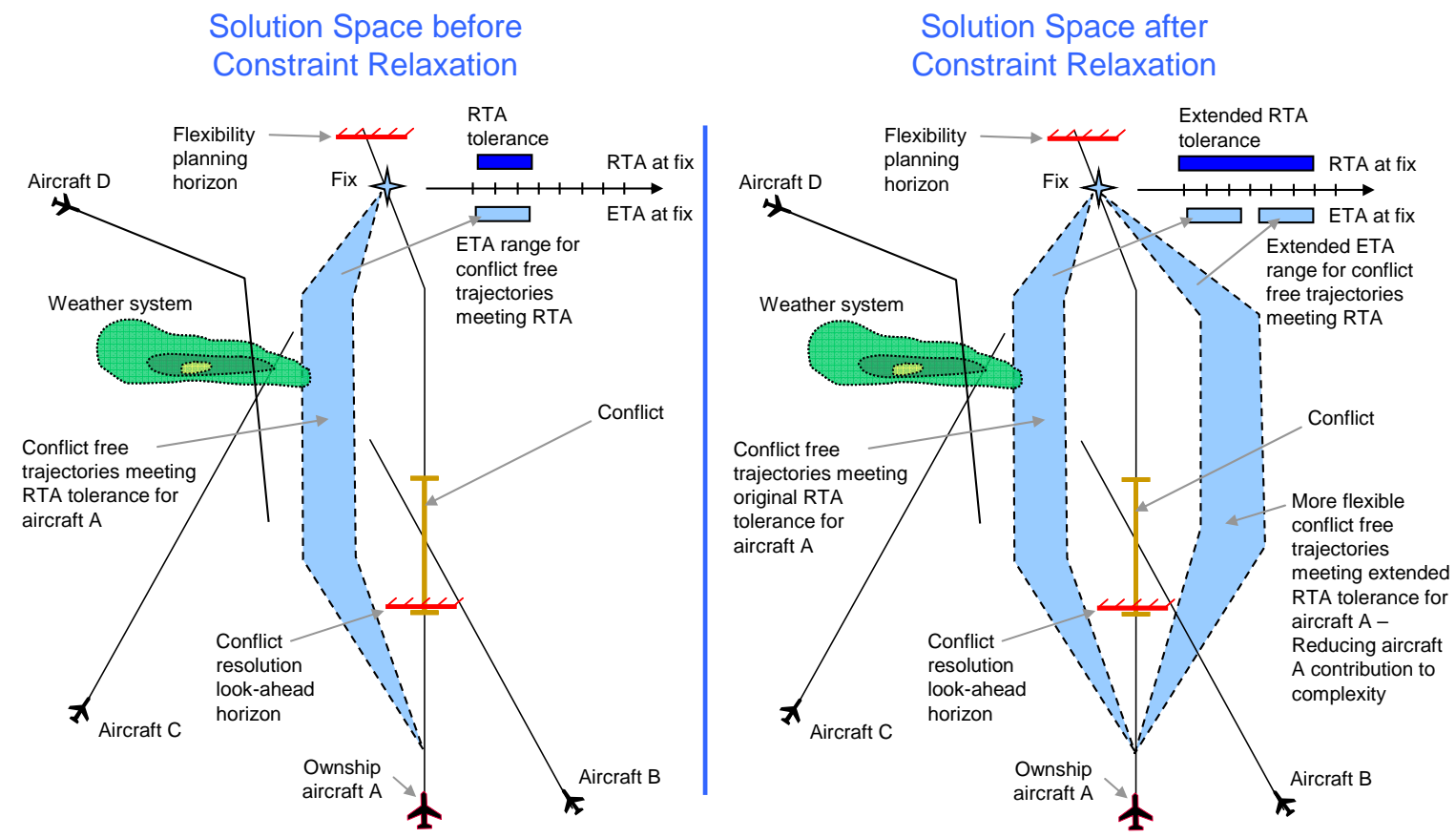

Figure 3 Constraint Minimization Example - Relaxing RTA Tolerance

\section{Analytical Framework and Definitions}

In order to develop metrics and methods for trajectory flexibility preservation and constraint minimization, these functions are posed in the framework of an aircraft trajectory solution space [14]. The trajectory is required to abide by a set of constraints imposed to achieve ATM objectives such as maintaining separation requirements and balancing demand and capacity. Therefore, these constraints define a solution space consisting of the set of feasible trajectories. Out of these trajectories an aircraft selects one that optimizes its preferences, such as minimizing fuel burn, delay, and passenger discomfort. This analysis considers limited-scope examples with only RTA and conflict constraints, in order to gain insight and establish a basis for generalization to other situations. The notion of trajectory flexibility is defined and the effects of the RTA and conflict constraints on it are highlighted.

\section{Trajectory Solution Space with RTA and Conflict Constraints}

A trajectory is represented by a 3-dimensional path (s) and a speed profile $(\mathrm{V}(\mathrm{s}))$ that determines the time $(\mathrm{t}(\mathrm{s}))$ at each location along the path. Using this representation Figure 4 depicts the trajectory solution space of an aircraft A flying towards a fix and facing constraints from multiple RTAs and a conflict with an intruder aircraft B (which may also represent a moving weather cell). Aircraft A may select from a number of paths $\mathrm{s}_{\mathrm{i}}$ (two of which, $\mathrm{s}_{0}$ and $s_{1}$, are analyzed in the figure) and speed profiles along each path. Figure 4 depicts, in an $\mathrm{s}_{\mathrm{i}}$ - $\mathrm{t}$ space, the solution space of aircraft $A$ along paths $s_{0}$ and $s_{1}$ given speed as the only degree of freedom along each path. The set of times that are reachable at a distance $\mathrm{s}_{\mathrm{i}}$ are bound by traveling at maximum speed $\mathrm{V}_{\max }$ and at minimum speed $\mathrm{V}_{\min }$. Along $\mathrm{s}_{0}$ the aircraft is required to meet $\mathrm{RTA}_{1}$ at distance $\mathrm{d}_{1}$ within a tolerance in time. The RTA constraint reduces the reachable set as shown in the figure by eliminating non-feasible regions, which consist of the reachable states that, if reached, the full speed range is not effective in meeting the RTA tolerance. These regions lie within lines with slopes $V_{\min }$ and $\mathrm{V}_{\max }$ extended from the RTA tolerance ends as described in [14]. In addition $\mathrm{s}_{0}$ is impacted by the intruder aircraft $B$ whose separation zone is expected to cross $s_{0}$ between distances $d_{3}$ and $d_{4}$. The geometry and timing of the conflict translates into an elliptical region in the $\mathrm{s}_{0}$-t domain with all points within corresponding to loss of separation. 

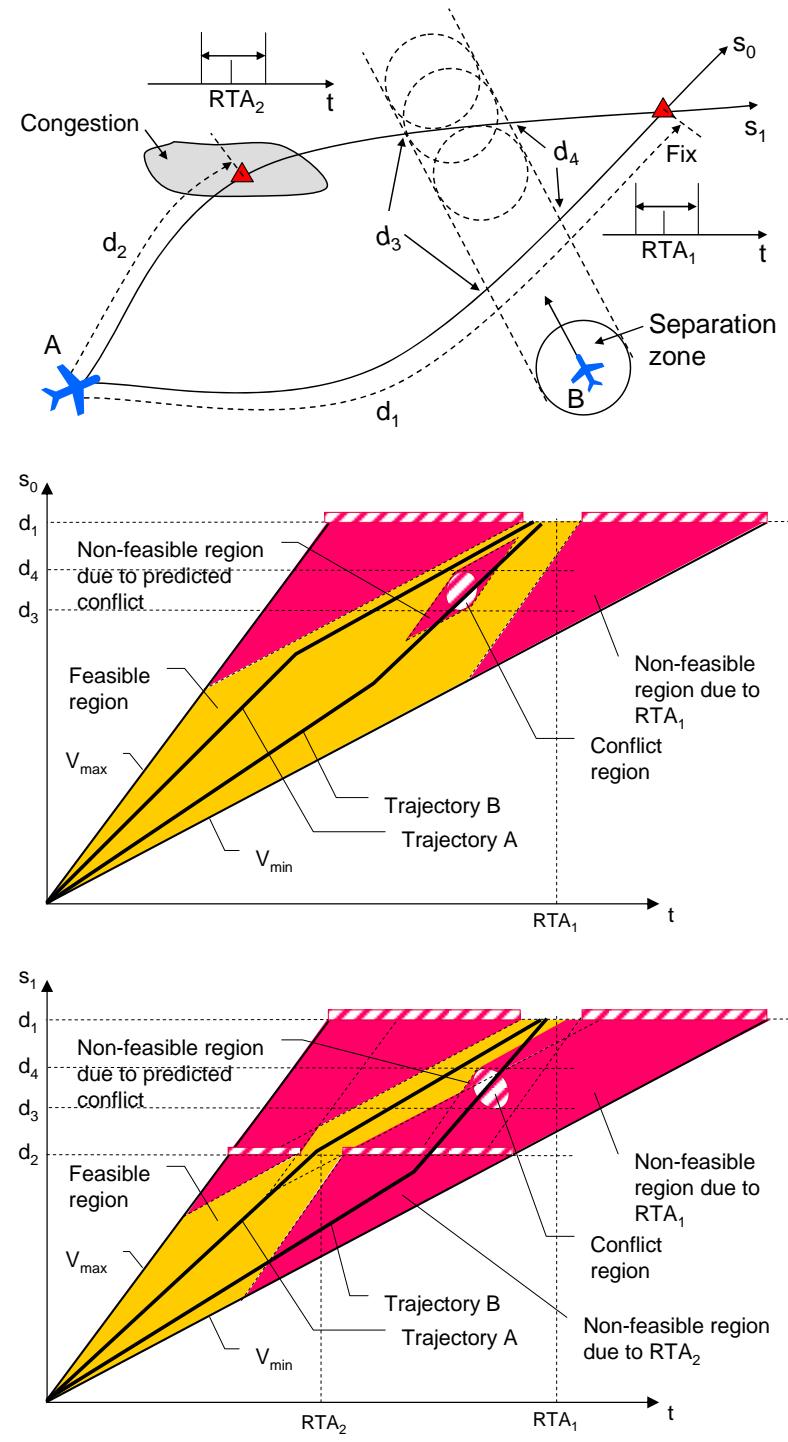

\section{Figure 4 Solution Space with RTA and Conflict Constraints}

A trajectory that crosses this region loses separation with the intruder and is hence infeasible. ${ }^{1}$ As shown in Figure 4 the conflict cuts out an additional infeasible region bound by the $\mathrm{V}_{\max }$ and $\mathrm{V}_{\text {min }}$ tangents to the elliptical conflict region [19]. Trajectory B is infeasible because of loss of separation with the intruder aircraft while trajectory $\mathrm{A}$ is feasible being conflict free and meeting $\mathrm{RTA}_{1}$.

\footnotetext{
${ }^{1}$ Idris et al. gives a mathematical formulation of the conflict region for a circular separation zone around an intruder aircraft moving at a constant speed [19]. In words: The separation zone occupies a line segment along $\mathrm{s}_{\mathrm{i}}$ that starts as a point when the zone first touches $\mathrm{s}_{\mathrm{i}}$, grows in size to the diameter length and shrinks to a point when the zone leaves the path $\mathrm{s}_{\mathrm{i}}$.
}

Imposing more constraints further limits the trajectory solution space of the aircraft. For example, along $\mathrm{s}_{1}$ the aircraft is required to meet two RTA constraints within tolerance: $\mathrm{RTA}_{2}$ at distance $\mathrm{d}_{2}$ because of a congestion region, and RTA $_{1}$ at $d_{1}>d_{2}$, in addition to the impact of the predicted conflict between $\mathrm{d}_{3}$ and $\mathrm{d}_{4}$. For convenience, the geometry in the figure is chosen such that $\mathrm{d}_{1}, \mathrm{~d}_{2}$, and $\mathrm{d}_{3}$ and equal along $\mathrm{s}_{0}$ and $\mathrm{s}_{1}$. As shown in Figure 4 the solution space is smaller than that along $\mathrm{s}_{0}$; trajectory $\mathrm{B}$ is infeasible because of loss of separation with the intruder aircraft or not meeting $\mathrm{RTA}_{2}$ while trajectory A remains feasible by meeting both RTAs and maintaining separation.

The locations and tolerances of $\mathrm{RTA}_{1}, \mathrm{RTA}_{2}$ or the conflict region in Figure 4, may leave no feasible trajectory that is conflict free and meets both RTAs. In this case the aircraft trajectory is over-constrained and requires relaxation of some constraints. Figure 4 demonstrates how relaxing a constraint by, for example, increasing an RTA tolerance opens up solution space and allows more feasible trajectories.

\section{Flexibility as Accommodation of Disturbances}

Disturbances may occur such as an additional traffic conflict that alter the images depicted in Figure 4 from what is predicted causing the aircraft to violate constraints. As was described in [14] the notion of trajectory flexibility is defined as the ability of the aircraft to accommodate such disturbances while abiding by the constraints. Disturbances may be classified into state or constraint disturbances: State disturbances result in aircraft state deviations from what is predicted by the trajectory. For example, imperfect wind forecast may be realized as a variation on ground speed and hence a variation on the aircraft state relative to the prediction over the prediction horizon. Constraint disturbances result in deviations in the constraints that define the aircraft trajectory solution space. They may be new constraints or modifications of currently imposed/predicted constraints. They include many types such as new TFM restrictions or new conflicts with traffic or weather cells, of which limited information is available at the prediction time. For example, the conflict or RTA 2 in Figure 4 may occur as disturbances that drastically changed the solution space. 
To better accommodate the possibility of such disturbances, an aircraft selects from a trajectory with sufficient flexibility. Two characteristics relevant to measuring flexibility have been identified: robustness and adaptability [14].

1. Robustness is defined as the ability of the aircraft to keep its planned trajectory ${ }^{2}$ unchanged in response to the occurrence of a disturbance. A trajectory that can withstand a disturbance without having to change is more robust than other trajectories that become infeasible when the disturbance occurs. In the context of the RTA/conflict constraint scenario of Figure 4 and considering the introduction of the conflict as a disturbance, a trajectory that remains feasible in terms of meeting the tolerances of both RTA $\mathrm{R}_{1}$ and $\mathrm{RTA}_{2}$ and avoiding the conflict despite the disturbance, which significantly reduced the solution space, is robust to this disturbance.

2. Adaptability is defined as the ability of the aircraft to change its planned trajectory in response to the occurrence of a disturbance that renders the current planned trajectory infeasible. A trajectory that positions the aircraft such that other feasible trajectories remain accessible to it if a disturbance occurred and rendered the current trajectory infeasible is more adaptable than another trajectory for which the disturbance leaves fewer or no feasible trajectories. In the context of the multiple RTA/conflict scenario of Figure 4, if trajectory B was selected it becomes infeasible when the conflict is predicted. The prediction of the conflict reduced the solution space. However, it left a set of trajectories for the aircraft that are feasible in terms of meeting both $\mathrm{RTA}_{1}$ and $\mathrm{RTA}_{2}$ and resolving the conflict. Therefore, the aircraft is able to adapt to this disturbance over a certain time, for example, by changing its planned trajectory from $\mathrm{B}$ to $\mathrm{A}$.

\section{Metrics and Analytical Example}

Selecting appropriate metrics for measuring flexibility in terms of its two characteristics, robustness and adaptability, requires generalization to a wide range of situations involving various degrees of freedom and types of disturbances.

\footnotetext{
${ }^{2}$ The robustness and adaptability characteristics apply to the full or part of a trajectory plan, such as a path or speed profile.
}

Towards this end, preliminary metrics are posed in the context of a simple scenario to gain insight and establish a basis to be generalized to more complex situations. Such generalization is subject of ongoing research and will be presented in future papers.

\section{Analysis of Solution Space in Simple Scenario}

The simple scenario involves a single aircraft selecting from a set of pre-specified paths to fly between its current position and a destination fix with the ability to vary speed along each path, as was depicted in Figure 4. The aircraft has to meet an RTA at the destination fix regardless of the selected path. Some paths pass through a congestion region which results in a second RTA constraint along these paths at the congestion region. The paths in the scenario may be impacted by one constraint disturbance: a predicted conflict with traffic that crosses the paths. State type disturbances are not considered, where the aircraft is assumed to fly its planned trajectory accurately.

The discrete path choice and the speed profile choice constitute a hierarchal decision process where it is assumed here that the aircraft selects a path first and then the speed profile to achieve its objectives. Once the aircraft selected the path, its only degree of freedom is selecting the speed profile along the path. The decision analyzed here is the selection of the path, where the only objective of the selection is to preserve (or maximize) flexibility (represented by the robustness and adaptability characteristics defined in the previous section) in accommodating the conflict prediction disturbance, using the speed degree of freedom. With these assumptions, the decision process is analyzed using initial definitions of metrics that measure robustness and adaptability of each path to the predicted conflict disturbance. It is important to note that this hierarchical decision process may not result in the most flexible trajectory (including path and speed profile). This is because the path is selected first based on aggregate flexibility metrics over the set of trajectories that the speed provides along each path. An integrated trajectory selection approach may result in a more optimal trajectory and will be addressed in future research.

In the context of this scenario, the solution space along each path is analyzed in terms of its flexibility to the prediction of the conflict with the 
intruder aircraft. Figure 5 depicts the solution space along a path $\mathrm{s}$ that is impacted by an RTA constraint at distance $d_{1}$ and a specific instance ${ }^{3}$ of the conflict prediction at a location prior to $\mathrm{d}_{1}$ and a time prior to $\mathrm{RTA}_{1}$ (as was analyzed in Figure 4). The conflict region divides the solution space into the following regions:

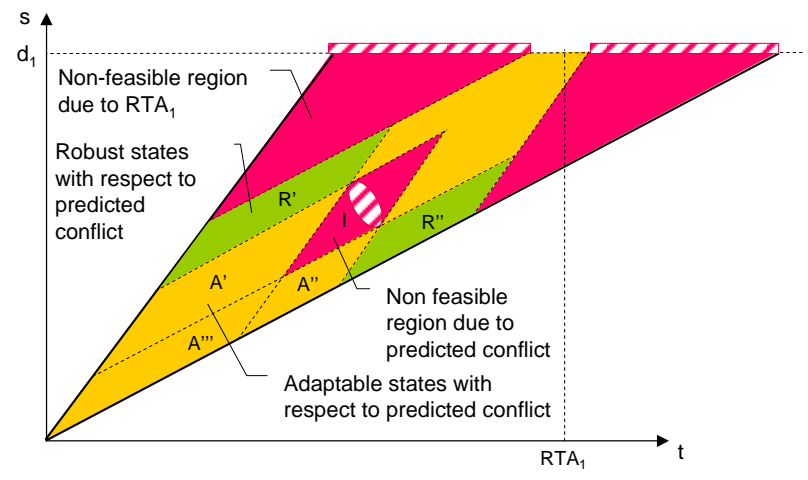

Figure 5 Robust and Adaptable States

1. Area I consists of the infeasible states which, once reached, violating the conflict constraint is unavoidable. These states cannot be part of a conflict free trajectory. This area is bound by the straight line tangents to the conflict region, with minimum and maximum speed slopes.

2. Area R consists of robust states which, once reached, conflict violation cannot occur however speed is varied. These states cannot be part of an infeasible trajectory with the predicted conflict. R may consist of multiple areas (R' and R" depicted in Figure 5).

3. Area A consists of adaptable states that may be part of either feasible or infeasible trajectories, with respect to the conflict. Area A is divided into multiple areas in Figure 5 indicating which area reaches the I and $\mathrm{R}$ states. The infeasible states, I, can be reached from all A', A" and A"' states. On the other hand, states R' can be reached from $\mathrm{A}^{\prime}$ and $\mathrm{A}^{\prime \prime}$ states, while states in $\mathrm{R}^{\prime \prime}$ can be reached from states in $\mathrm{A}^{\prime \prime}$ and $\mathrm{A}^{\prime \prime}$.

\section{Robustness Metric}

The robustness of an aircraft planned trajectory to a disturbance is measured by the probability that

\footnotetext{
${ }^{3}$ Other instances correspond, for example, to variability in the intruder aircraft trajectory prediction.
}

the trajectory stays feasible (by not violating any constraint) if the disturbance occurred. The robustness of the path depicted in Figure 5, which is a partial trajectory plan, may be measured by the probability that an arbitrarily selected trajectory along the path (i.e. a speed profile) falls in the feasible area R, as opposed to the infeasible area I, after the prediction of the conflict. Assuming an equally likely trajectory selection, one metric (RBT) that measures this probability is the ratio of the number of feasible trajectories that fall in the $\mathrm{R}$ area (i.e., despite the disturbance) to the total number of feasible trajectories along the path (i.e., independent of the disturbance):

RBT (given disturbance) $=$ Number of feasible trajectories (given disturbance) / Total number of feasible trajectories (without disturbance).

Computing this metric requires estimating the total number of trajectories abiding by all constraints except the predicted conflict and the number of feasible trajectories with respect to the predicted conflict. For illustration, an example is given in Figure 6 with the additional constraints that only a single change of speed is allowed along the path and with a single point (zero tolerance) RTA. The solution space that meets RTA $_{1}$ independently from the predicted conflict is bound as before by the minimum and maximum speed lines extended from the point $\left(\mathrm{RTA}_{1} \mathrm{~d}_{1}\right)$ and the current state. With the additional constraints each point in this space corresponds to the speed change location of a single trajectory, and each trajectory that meets the RTA changes its speed at one point in this space.

Therefore, the number of trajectories that meet the RTA (independently form the predicted conflict disturbance) corresponds exactly to the area of the solution space (S). The analysis of this solution space into areas R, I and A with respect to the predicted conflict is depicted in the figure. The infeasible area I is bounded by the two singlespeed-change trajectories that are tangent to the predicted conflict region and end at $\mathrm{RTA}_{1}$. Correspondingly the areas $\mathrm{R}^{\prime}$ and $\mathrm{R}$ " are bound by these lines and the minimum-/maximum-speed boundaries of the solution space that meets $\mathrm{RTA}_{1}$. Area R' can be reached from area A' and area R" can be reached from area $A^{\prime \prime}$. The number of feasible trajectories that fall in $\mathrm{R}$ is measured by the area of the $\mathrm{R}$ region, because any speed change 
outside R corresponds to an infeasible trajectory, and each point in the area $\mathrm{R}$ corresponds to the location of one possible speed change and hence exactly to a single feasible trajectory. Therefore, $\mathrm{RBT}=\left(\mathrm{R}^{\prime}+\mathrm{R}^{\prime \prime}\right) / \mathrm{S}$.

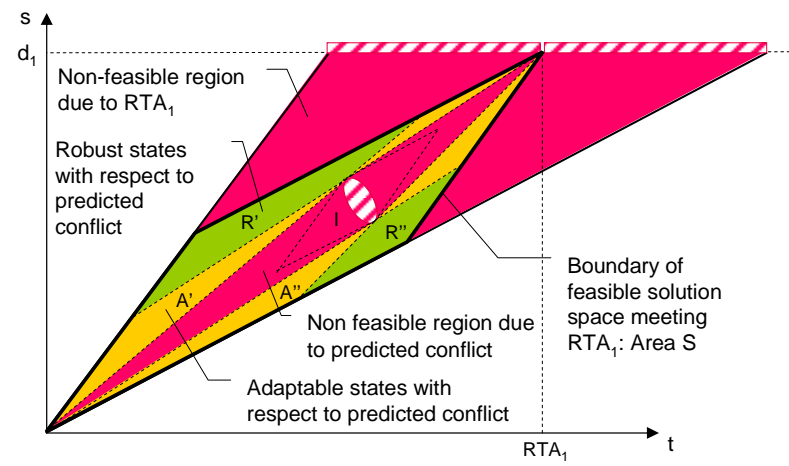

Figure 6 Solution Space with One Speed Change

Then, assuming a stochastic behavior of the intruder aircraft of which the predicted conflict situation depicted in Figure 5 or Figure 6 is one instance ' $i$ ', the metric is averaged over the instances. For example, if the intruder stochastic behavior is characterized by a distribution $\mathrm{P}_{\mathrm{i}}$ over instances $\mathrm{i}: 1-\mathrm{N}$, then the robustness metric is integrated over the instances: $\Sigma_{\mathrm{i}: 1-\mathrm{N}}\left[\mathrm{P}_{\mathrm{i}} \times \mathrm{RBT}_{\mathrm{i}}\right]$.

\section{Adaptability Metric}

A planned trajectory that falls in the infeasible area I in Figure 5 should be adapted by changing it to a feasible trajectory that contains states in area R. This adaptation is meaningful only for trajectories that contain infeasible states in I and is possible as long as the current state is an adaptive state in area A. Therefore, the adaptability of the path is dictated by the availability of area $R$ and how reachable it is from states in area A, using the speed degree of freedom. Therefore, one metric that measures adaptability (ADP) is the absolute number of feasible trajectories that fall in area $\mathrm{R}$ :

$A D P$ (Given disturbance $)=$ Number of feasible trajectories (given disturbance).

Therefore, for the example depicted in Figure 6 , adaptability is measured by the area $\mathrm{R}$ : ADP $=$ $\mathrm{R}^{\prime}+\mathrm{R}$ ". Then, assuming a stochastic behavior of the intruder aircraft characterized by a distribution $P_{i}$ over conflict instances $\mathrm{i}: 1-\mathrm{N}$, the metric is averaged over the instances as described for the robustness metric: $\Sigma_{\mathrm{i}: 1-\mathrm{N}}\left[\mathrm{P}_{\mathrm{i}} \times \mathrm{ADP}_{\mathrm{i}}\right]$.

The robustness and adaptability metrics proposed are used in this scenario to compare flexibility among different paths by measuring the set of feasible trajectories that the speed degree of freedom provides along each path. This comparison is used to make a path selection based on properties aggregated over the set of trajectories along the path. The flexibility metric is ultimately used to plan a full trajectory including the path and the speed profile. While this integrated planning process is outside the scope of this paper, it is important to note that the robustness and adaptability metrics proposed can be extended to the integrated planning by maintaining or preserving their values at each step along a trajectory. For example, adaptability decreases as the aircraft moves along a trajectory because the number of feasible trajectories decreases. This can be seen from Figure 5, where the states in area A"' are more adaptable having access to both $\mathrm{R}^{\prime}$ and $\mathrm{R}^{\prime \prime}$, while states in areas $\mathrm{A}^{\prime}$ and $\mathrm{A}^{\prime \prime}$ are less adaptable having access only to $\mathrm{R}^{\prime}$ or $\mathrm{R}^{\prime \prime}$ respectively. Hence, adaptability decreases continuously as the aircraft proceeds along a trajectory transitioning through $\mathrm{A}^{\prime \prime}$ and then from $\mathrm{A}$ "' to either $\mathrm{A}^{\prime}$ or A". An adaptable trajectory may be planned by minimizing the rate at which such reduction in adaptability occurs along the trajectory. Extending the path decision presented here to complete integrated trajectory planning based on preserving flexibility will be presented in future papers.

\section{Decision Analysis using Suggested Metrics}

Two path selection cases are analyzed, case 1 demonstrating the potential impact of preserving flexibility on traffic complexity and case 2 the potential impact of relaxing constraints on preserving flexibility.

Case 1 is presented in Figure 7: An aircraft is deciding to path stretch to meet an RTA constraint at a destination fix. It compares two path stretches; $\mathrm{s}_{1}$ which infringes on a traffic flow in an opposing direction and $s_{2}$ which infringes on a traffic flow in an aligned direction. The aircraft selects one based on its flexibility using the speed degree of freedom only to avoid the disturbance of a potential conflict. For illustration, the predicted conflict geometry and 

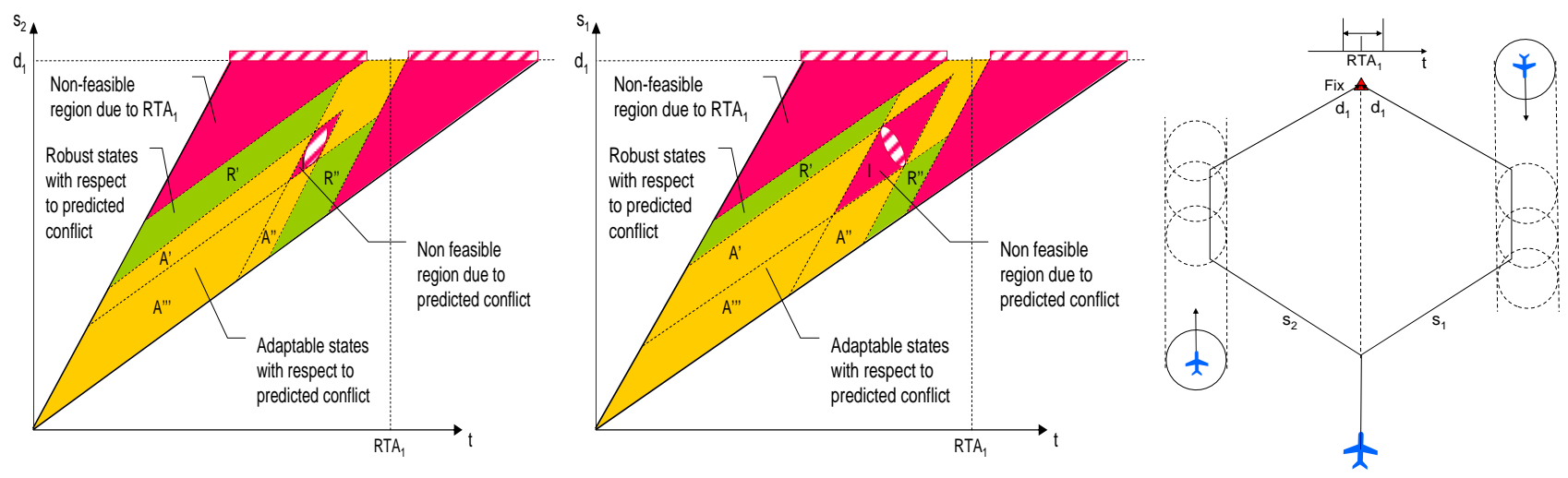

Figure 7 Case 1: Aligned versus Opposing Traffic

timing are selected such that the conflict regions along the two paths are exactly symmetric about the vertical axis [19]. Figure 7 shows visually, for a specific conflict prediction instance, that the R/I areas (including $\mathrm{R}^{\prime}$ and $\mathrm{R}^{\prime \prime}$ ) are significantly larger/smaller for path $\mathrm{s}_{2}$ aligned with the traffic relative to path $\mathrm{s}_{1}$ opposing the traffic. Assuming a direct relationship between the $\mathrm{R} / \mathrm{I}$ areas and the number of feasible/infeasible trajectories, as was illustrated in the example in Figure 6, the robustness (relative number of feasible trajectories) and adaptability (absolute number of feasible trajectories) are higher for $s_{2}$ than $s_{1}$. Everything else being equal, including identical stochastic behavior of the intruder traffic for both paths, the robustness and adaptability metrics are more favorable for $s_{2}$ than $s_{1}$. Therefore, the aircraft would decide on the path stretch which is aligned with traffic. This shows that the aircraft's decision based on preserving its flexibility (represented by the robustness and adaptability metrics suggested in this example and using only speed) results in aligning the aircraft with other traffic, and hence reducing its contribution to complexity. This hypothesis will be tested under more rigorous and general scenarios to be published in later papers.

Case 2 is presented in Figure 8: An aircraft is deciding to path stretch to meet an RTA constraint $\left(\mathrm{RTA}_{1}\right)$ at a destination fix. It compares two path stretches $s_{1}$ and $s_{2}$ both infringing on a traffic flow in an opposing direction, such that the conflict prediction disturbance is identical between the two paths. However, path stretch $\mathrm{s}_{2}$ passes through a congestion region resulting in $\mathrm{RTA}_{2}$ at an intermediate location. Figure 8 shows that the $\mathrm{RTA}_{2}$ constraint reduces the solution space considerably ( $R$ area relative to I area) for $s_{2}$ relative to $s_{1}$ resulting in lower robustness and adaptability to the conflict prediction disturbance. This example demonstrates that minimizing constraints, for example by removing $\mathrm{RTA}_{2}$ or increasing its tolerance, results in higher flexibility. This hypothesis will be tested under more rigorous and general scenarios to be published in later papers.

\section{Concluding Remarks and Future Research}

This paper described a trajectory-oriented
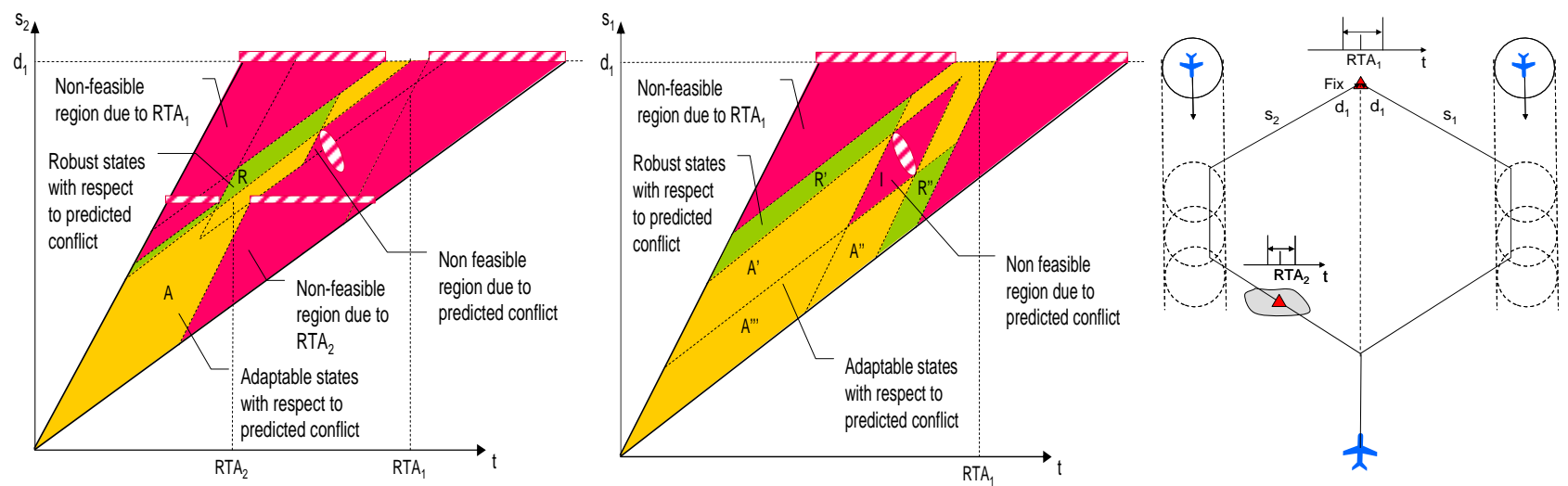

Figure 8 Case 2: One versus Two RTA Constraints 
approach to managing traffic complexity: A trajectory flexibility preservation function enables an aircraft to preserve flexibility in accommodating disturbances from, for example, other traffic and weather activity. A trajectory constraint minimization function enables ground-based agents, in collaboration with air-based agents, to impose just-enough constraints on trajectories to achieve ATM objectives, such as separation assurance and flow management. The concept hypothesizes that by each aircraft autonomously preserving its own trajectory flexibility, aggregate system objectives, such as maintaining acceptable traffic complexity, are naturally achieved. It also hypothesizes that minimizing the constraints imposed on a trajectory, without jeopardizing the intended ATM objectives, increases its flexibility. Towards testing these hypotheses an analytical framework for defining metrics and developing methods to preserve trajectory flexibility and minimize its constraints was presented. In this framework flexibility was defined in terms of robustness and adaptability to disturbances and metrics were derived in the context of a simple scenario. The scenario involved the selection a predefined path with limited degrees of freedom, namely speed variation along the path, in simple constraint situations involving meeting multiple times of arrival and resolving a conflict. The notions and metrics, described conceptually and qualitatively in this paper, will be formalized and extended to other degrees of freedom and more general constraints/disturbance situations in future research. Some of these extensions include:

\section{Multiple Degrees of Freedom and Constraint Disturbances}

While the definitions of robustness and adaptability and their relationship to the trajectory solution space are generic, formulation and algorithms to exercise them with different degrees of freedom may vary. Therefore, the analyses and metrics developed in this paper for selecting a predefined path while varying speed will be generalized to other degrees of freedom. The analysis and metrics will also be generalized to situations involving other types and combinations of constraints and disturbances, and their uncertainties. The multiplicity of the constraints and their types also gives rise to a prioritization among them, which is important when the aircraft is unable to meet all of the constraints. For example, if the aircraft is over-constrained, it may report to the ground-based traffic manager that it is unable to meet an RTA because of the conflict. In this case the traffic manager may relax the RTA ensuring safety at the expense of TFM objectives.

\section{Tradeoff between Robustness, Adaptability and Other Objectives}

In some situations a tradeoff between robustness and adaptability may exist. This tradeoff depends on the decision maker's risk attitude. For example, a conservative decision maker may favor robustness to minimize having to accommodate the disturbance. A more risk prone attitude may tolerate a certain chance of dealing with the disturbance as long as there is sufficient adaptability.

Flexibility was defined in this paper as the aircraft ability to accommodate disturbances while abiding by all constraints. This goal is traded with other objectives of the aircraft such as fuel efficiency. Such objectives may be formulated as constraints on the trajectory solution space and treated in the flexibility preservation problem, or as objectives that compete with the flexibility preservation objective.

\section{Aggregate Cooperative Behavior}

The hypothesis made is that the flexibility preserving behavior of each individual aircraft will naturally result in less complex traffic situations. This hypothesis will be tested in future research by prototyping and experimenting the metrics and methods proposed in this paper. If the hypothesis is true then distributed flexibility preservation results in implicit coordination between aircraft. However, some level of explicit coordination may be needed to mitigate complexity. For example, information sharing between aircraft may be needed to coordinate the distributed flexibility preservation actions. This information may be used to model the disturbance uncertainties such as those associated with the conflict prediction analyzed in this paper.

\section{References}

[1] Joint Planning and Development Office, "Next Generation Air Transportation System Integrated 
Plan," URL:

http://www.jpdo.gov/library/NGATS_v1_1204r.pdf

[2] Wing, D., 2005, “A Potentially Useful Role for Airborne Separation in 4D-Trajectory ATM

Operations," AIAA-2005-7336.

[3] NASA, 2004, "DAG-TM Concept Element 5 En Route Free Maneuvering for User-Preferred Separation Assurance and Local TFM Conformance Operational Concept Description," AATT Project Milestone 8.503.10, NASA Airspace Systems Program Office, Washington D.C.

[4] NASA, 2004, "DAG-TM Concept Element 11 Terminal Arrival Self-Spacing for Merging and InTrail Separation Operational Concept Description," AATT Project Milestone 8.652.7, NASA Airspace Systems Program Office, Washington D.C.

[5] Hill, J.C., J.K. Archibald, W.C. Stirling, and R.L. Frost, 2005, "A Multi-Agent System Architecture for Distributed Air Traffic Control," AIAA-2005-6049.

[6] Wollkind, S., J. Valasek, and T. Ioeger, 2004, "Automated Conflict Resolution for Air Traffic Management Using Cooperative Multiagent Negotiation," AIAA-2004-4992.

[7] Versteegt, H.H., and H.G. Visser, 2002, "Traffic Complexity Based Conflict Resolution," AIAA2002-4443.

[8] Ballen, M., V. Sharma, R. Vivona., E. Johnson, and E. Ramiscal, 2002, "A Flight Deck Decision

Support Tool for Autonomous Airborne Operations," AIAA-2002-4554.

[9] Mondoloni, S., and S. Conway, 2001, “An Airborne Conflict Resolution Approach Using A Genetic Algorithm," AIAA-2001-4054.

[10] Vivona, R., D. Karr, and D. Roscoe, 2006, "Pattern-Based Genetic Algorithm for Airborne Conflict Resolution," AIAA-2006-6060.

[11] Barhydt, R., and P. Kopardekar, 2005, "Joint NASA Ames/Langley Experimental Evaluation of Integrated Air/Ground Operations for En Route Free maneuvering," $6^{\text {th }}$ USA/Europe ATM R\&D Seminar, Baltimore, MD.

[12] Krishnamurthy, K., B. Barmore, and F. Bussink, 2005, “Airborne Precision Spacing in
Merging terminal Arrival Routes," $6{ }^{\text {th }}$ USA/Europe ATM R\&D Seminar, Baltimore, MD.

[13] Mediterranean Free Flight Programme Final Report, 2005, D821, http://www.medff.it/public/index.asp.

[14] Idris H., D. Wing, R. Vivona, and J.L. GarciaChico, 2007, "A Distributed Trajectory-Oriented Approach to Managing Traffic Complexity," AIAA-2007-7731.

[15] Delahaye, D., S. Puechmorel, R.J. Hansman, and J. Histon, 2003, "Air traffic complexity based on non linear dynamical systems," $5^{\text {th }}$ USA/Europe ATM R\&D Seminar, Budapest, Hungary.

[16] Kopardekar. P. and S. Magyarits, 2003, "Measurements and prediction of dynamic density," $5^{\text {th }}$ USA/Europe ATM R\&D Seminar, Budapest, Hungary.

[17] Riley, V., G. Chatterji, W. Johnson, R. Mogford, P. Kopardekar, E. Sierra, and G. Lawton, 2004, "Pilot perceptions of airspace complexity, part 2," DASC.

[18] Erzberger, H., T.J. Davis, and S.M. Green, 1993, "Design of Center-TRACON Automation System," AGARD Meeting on Machine Intelligence in ATM, Berlin, Germany.

[19] Idris, H., T. Hsu, R. Vivona, and S. Green, 2003, "Time Based Conflict Resolution Algorithm and Application to Descent Conflicts," AIAA-20035517.

\section{Acknowledgements}

This research was funded by NASA under contract NNA07BA86C.

\section{Email Addresses}

Husni.idris@L-3com.com;

David.wing@nasa.gov

26th Digital Avionics Systems Conference October 21, 2007 